\title{
Preferential Loss of Striato-External Pallidal Projection Neurons in Presymptomatic Huntington's Disease
}

\author{
Roger L. Albin, MD,* Anton Reiner, PhD, $\uparrow$ Keith D. Anderson, PhD, $\dagger$ Leon S. Dure IV, MD,* \\ Barbara Handelin, PhD, $\ddagger$ Rosemary Balfour, PhD, $;$ William O. Whetsell, Jr, MD,, \\ John B. Penney, MD,* and Anne B. Young, MD, PhD*
}

We have reported previously that striatal projection neurons are differentially affected in the course of Huntington's disease, and in a prior patient report we noted that differential loss of striatal projection neurons occurs also in patients with presymptomatic Huntington's disease. Striatal neurons projecting to the external segment of the globus pallidus or the substantia nigra show evident loss, whereas those projecting to the internal segment of the globus pallidus appear relatively spared at presymptomatic and early stages of symptomatic Huntington's disease. We now report similar findings in a second apparently presymptomatic Huntington's disease allele carrier.

Albin RL, Reiner A, Anderson KD, Dure LS IV, Handelin B, Balfour R, Whetsell WO, Jr, Penney JB, Young AB. Preferential loss of striato-external pallidal projection neurons in presymptomatic Huntington's disease. Ann Neurol 1992;31:425-430

The histopathological hallmark of Huntington's disease (HD) is pronounced atrophy of the striatum, especially the caudate nucleus. Within the striatum, there is differential involvement of subpopulations of striatal neurons. Striatal interneurons cocontaining the peptides somatostatin and neuropeptide $Y$ and the enzyme NADPH-diaphorase appear to be spared in patients with HD $\{1,2]$. Similarly, the perikarya of striatal cholinergic interneurons appear also to be spared [3, 4], though there is an impressive loss of striatal choline acetyltransferase activity and of cholinesterase staining within the striatum $[5,6]$. These classes of striatal interneurons comprise only a small fraction of all striatal neurons. The overwhelming majority of striatal neurons are projection neurons, and striatal projection neurons are markedly depleted in the course of HD. Striatal projection neurons are subdivided into separate populations based on their projection targets and neuropeptide content [7-13]. Striatal neurons projecting to the external segment of the globus pallidus (GPe) are enriched in enkephalins (ENK), whereas striatal neurons projecting to the internal segment of the globus pallidus (GPi) or to the substantia nigra (SN) are enriched in substance $P(S P) ~[12,13]$. We have shown previously that ENK-containing striatal neurons projecting to the GPe and SP-containing stria- tal neurons projecting to the SN are preferentially depleted in early grade HD with relative preservation of SP-containing striatal neurons projecting to the GPi [14]. By late grade HD, all striatal projection neurons show extensive loss. This sequence of striatal projection neuron loss is correlated with the evolution of some clinical features of $\mathrm{HD}[14,15]$. We have also reported abnormalities of striatal projection neurons similar to those of early grade HD in a presymptomatic carrier of the HD allele [16]. We now report preferential loss of striato-external pallidal projection neurons in a second presymptomatic carrier of the HD allele.

\section{Case Report and Methods \\ Case Report}

The subject was a man from a family with several individuals afflicted with typical Huntington's disease. His birth, development, adolescence, and early adulthood were apparently normal. An excellent athlete, he was offered and declined a college baseball scholarship. After graduation from high school, he served in the military for 4 years and was honorably discharged. He subsequently went into the roofing business with one of his relatives. Two to 3 years before his death, family and friends noticed changes in his personality. Formerly a pleasant and agreeable individual, he became unpredictably temperamental and argumentative, and sometimes physically violent. He began to use over-the-counter
From the *Department of Neurology, University of Michigan, Ann Arbor, MI; ${ }^{\circ}$ Department of Anatomy and Neurobiology, University of Tennessee, Health Science Center, Memphis; Integrated Genetics, Framingham, MA, and \$Department of Pathology, Vanderbilt University, Nashville, TN
Received May 16, 1991, and in revised form Aug 22. Accepted for publication Sep 26, 1991.

Address correspondence to Dr Albin, Neuroscience Laboratory Building, 1103 E. Huron, Ann Arbor, MI 48104 
stimulants frequently but did not abuse illicit drugs. He continued to work as a roofer until the time of death and his ability to work was unimpaired. Specifically, there was no clumsiness, incoordination, or decrease in his rate of work. No adventitious movements were noted by friends or family members. His father did notice that he had become somewhat fidgety and had difficulty sitting still. No relative, including those involved in the care of family members suffering from $\mathrm{HD}$, noticed any movements that resembled chorea. At the age of 28 years, in the course of an argument, he was killed by a gunshor to the trunk.

After appropriate clearances, an autopsy was performed approximately 20 hours after death. The brain was removed in the routine fashion and hemisected in the midsagittal plane. One-half was fixed in $10 \%$ neutral buffered formalin and the other one-half frozen to $-80^{\circ} \mathrm{C}$ immediately after removal. After 14 days of fixation, the formalin-fixed half was cut up into $1-\mathrm{cm}$-thick coronal sections and selected tissue blocks were prepared for routine light microscopic study. Blocks through the pallidum and nigra were used for immunohistochemical analysis after 6 additional months in fixative.

Neuropathological analysis was performed by one of the authors (W.O.W.) several months before assessment of HD allele status. In a thorough survey of the cerebral cortex, subcortical white matter, basal ganglia, thalamus, midbrain, pons, medulla, and cerebellum, there were no significant neuropathological abnormalities. Specifically, there was no evidence of caudate or cerebral cortical atrophy, no obvious loss of striatal neurons, and no striatal or cortical gliosis. Masson trichrome-stained sections confirmed the absence of striatal gliosis.

\section{Methods}

LINKAGE ANALYSIS. Most members of this pedigree were examined for evidence of HD by two of the authors (R.L.A. and L.S.D.) and blood samples were drawn for use in linkage analysis. DNA was extracted from the blood samples of membars and from a frozen block of cerebellar cortex of the subject using standard techniques. The entire family study, including extraction of DNA from brain and blood samples, was performed in duplicate by two independent technicians. Southern blots were generated using appropriate restriction enzymes for the following informative polymorphic DNA markers that are known to be closely linked to the HD gene: pKO82/HindIII, YNZ32/Pst, and BS674/Act. The resulting genotypes at these markers for each relevant member of the family were coded into the MLINK function of the LINKAGE program [17]. No recombination events were observed among the nine informative meiotic events scored. All markers were conservatively placed at $2 \%$ recombination distance from the HD locus; these values for theta were combined with ages of onset and liability classes for each family member to calculate an assessment of accuracy for gene-carrier status of the subject.

IMMUNOHISTOCHEMISTRY. The integrity of specific types of striatal projection neurons was examined by immunohistochemical staining of their terminals for ENK and SP in coronal sections of the pallidum and substantia nigra. Well-characterized antisera directed against Leu-enkephalin (dilution, 1:500; INCstar, Stillwater, MN), met-enkephalinArg ${ }^{6}-$ Gly $^{\top}-$ Leu $^{8}$ (dilution, 1:500; gift of Dr G. J. Dockray), and SP (dilution, 1:1,000; Accurate Chemical and Scientific, Westbury, NY) were used, and immunohistochemistry was performed using the peroxidase-antiperoxidase techniciue as previously described [14]. Striatal gliosis was assessed with glial fibrillary acidic protein (GFAP) immunoreactivity using a well-characterized antiserum (INCstar; 1/500).

The extent of the reduction of immunohistochemically stained fibers in GP and SN was determined with quantitative image analysis, using a Newvicon camera, video capture board, MacIIci computer, and sofrware called Image (developed by Wayne Razband, National Institutes of Health, Bethesda, MD). Thresholding techniques [18] were used to measure fiber abundance in the presymptomatic brain compared with control subjects. Nine fields of the GPe, 18 fields of the GPi, and five fields of the nigra of a single section of the presymptomatic subject and control sections matched for level of the neuraxis were viewed with a $40 \times$ objective, and the gray scale adjusted to maximize contrast between labeled fibers and unstained background. Gray scale thresholds were set to "extract" labeled fibers by removing pixels with gray scale values between the thresholds from the image. The area of the removed pixels was computed and fiber abundance expressed as the area of extracted pixels divided by the total area of the field. ENK-immunoreactive (IR) fiber abundance in the GPe and SP-IR fiber abundance in GPi and nigra were compared with fiber abundance determinations in four control subjects matched for time in fixative. Time in fixative is a crucial variable affecting the intensity of staining of striatal efferent fibers (A. Reiner and colleagues, unpublished data). Threshold analysis of GPe ENK-IR fiber abundance and SP-IR fiber abundance in the GPi and nigra was also performed on the previously reported presymptomatic specinen and three additional control subjects

\section{Results}

Linkage Analysis

Results of the linkage analysis revealed a $97 \%$ probability that the subject was a carrier of the HD allele.

\section{Immunobistocbemistry}

GFAP staining of the striatum revealed no increase in GFAP immunoreactivity compared with control striatum.

In normal pallidum, dendrites of pallidal neurons are coated with the intensely immunoreactive terminals of striatopallidal neurons, giving the pallidal dendrites the so-called woolly fiber appearance [19] (Figs 1, 2). In the pallidum of the presymptomatic individual, SP-IR woolly fibers in the GPi were normal in appearance, but there was a reduction in the number of terminals of ENK-IR fibers in the GPe (see Figs 1, 2). A slight loss of SP-IR fibers was observed in the $\mathrm{SN}$ of the presymptomatic individual (see Figs 1,2). Threshold analysis of fiber abundance in the presymptomatic individual confirmed these observations. SP-IR fiber abundance in the GPi was $94.4 \%$ of control; ENK-IR fiber 


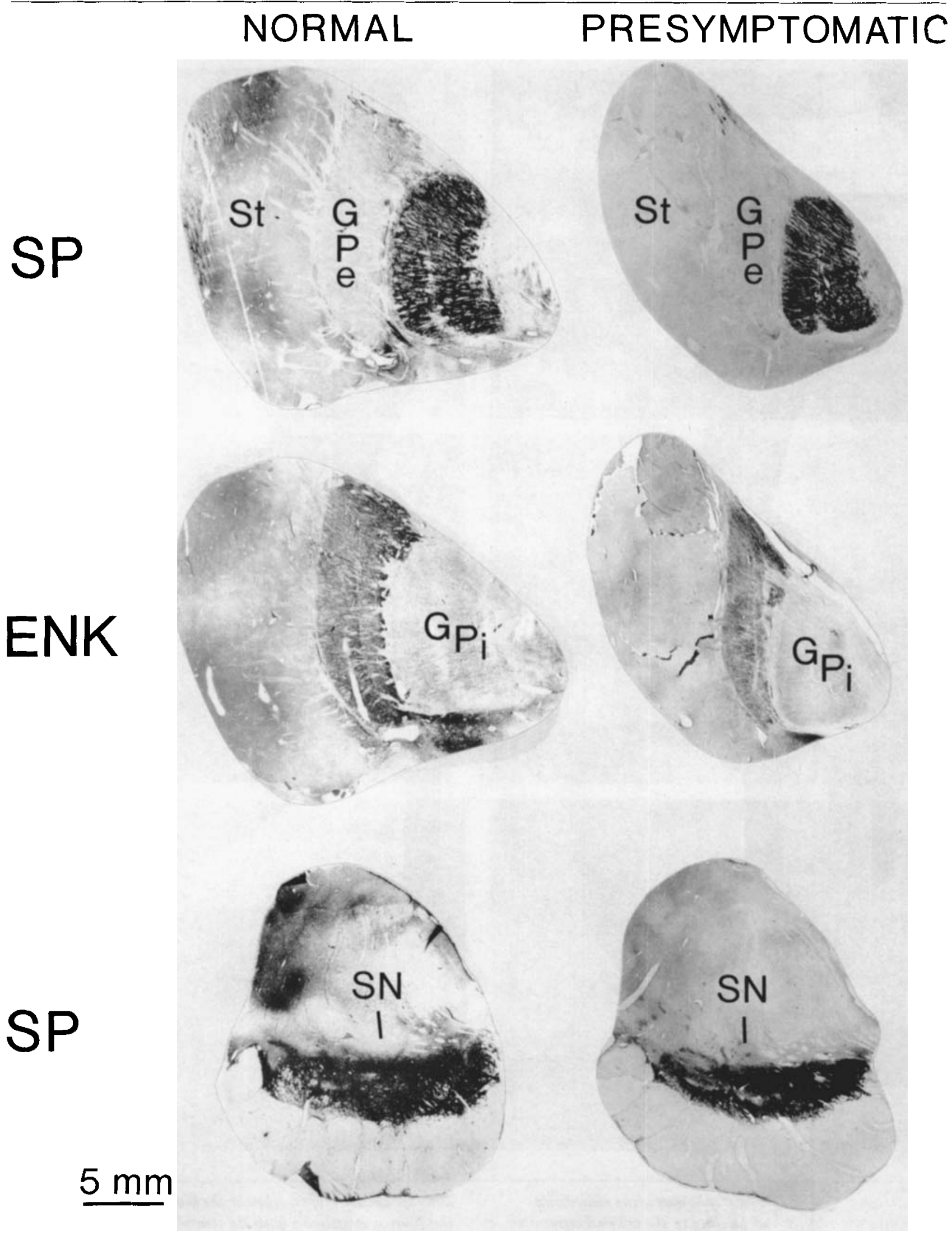

Fig 1 . The pallidum and substantia nigra of the presymptomatic subject and a control specimen. Substance P-immunoreactivity (SP) in the internal segment of the globus pallidus (GPi) of the presymptomatic is indistinguishable from normal but sligbtly reduced in the substantia nigra (SN). Enkephalinimmunoreactivity (ENK) in the external segment of the globus pallidus $(G P e)$ of the presymptomatic subject is also less than normal. The control specimen and presymptomatic specimen sections are at similar levels of the neuraxis and the pallidum of the presymptomatic specimen appears atropbic. The existence of pallidal (or nigral shrinkage) cannot be assessed without volumetric measurements. 


\section{NORMAL}
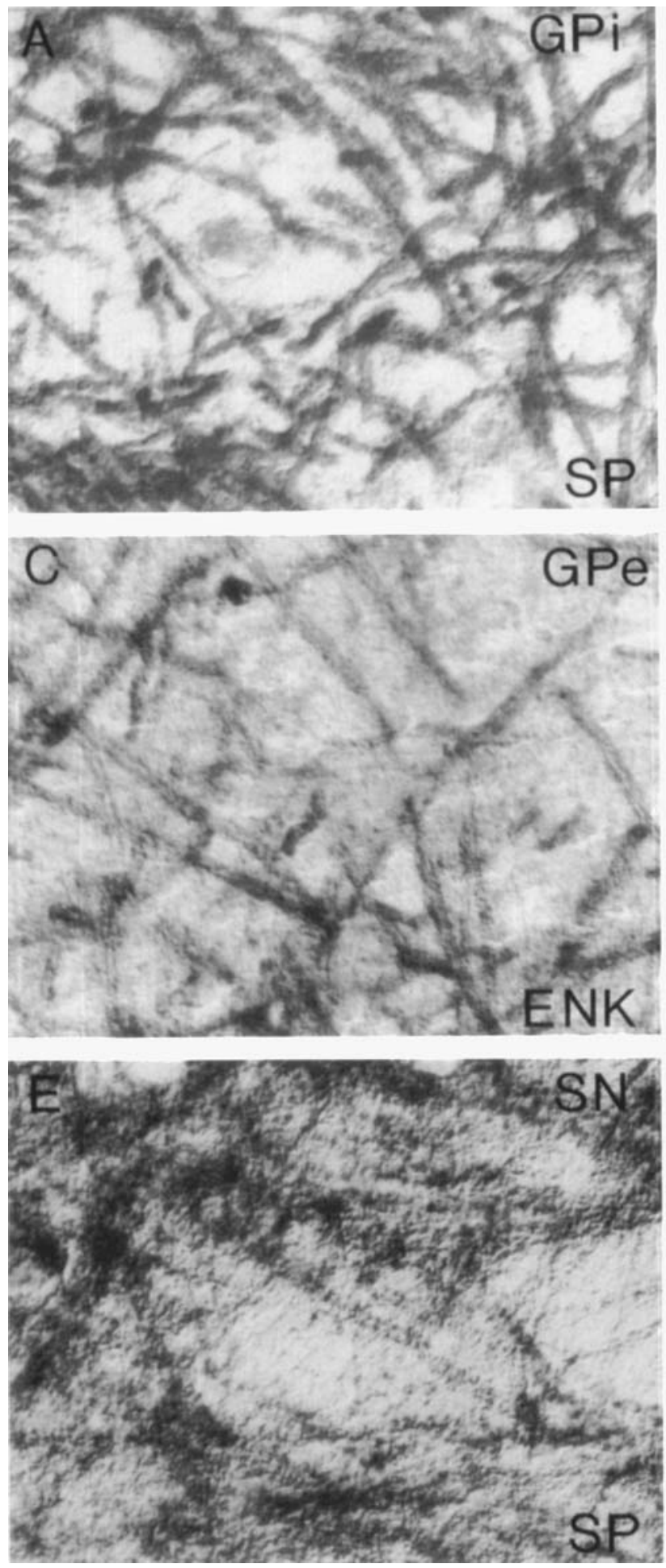

Fig 2. High-power photomicrographs comparing enkephalinimmunoreative (ENK) fiber labeling in the external segment of the globus pallidus ( $G P$ e) with substance $P$-immunoreactive (SP) fiber staining in the internal segment of the globus pallidus (GPi) in the subject of this report and a control subject. The density of ENK-immunoreactive fibers is reduced when compared
PRESYMPTOMATIC
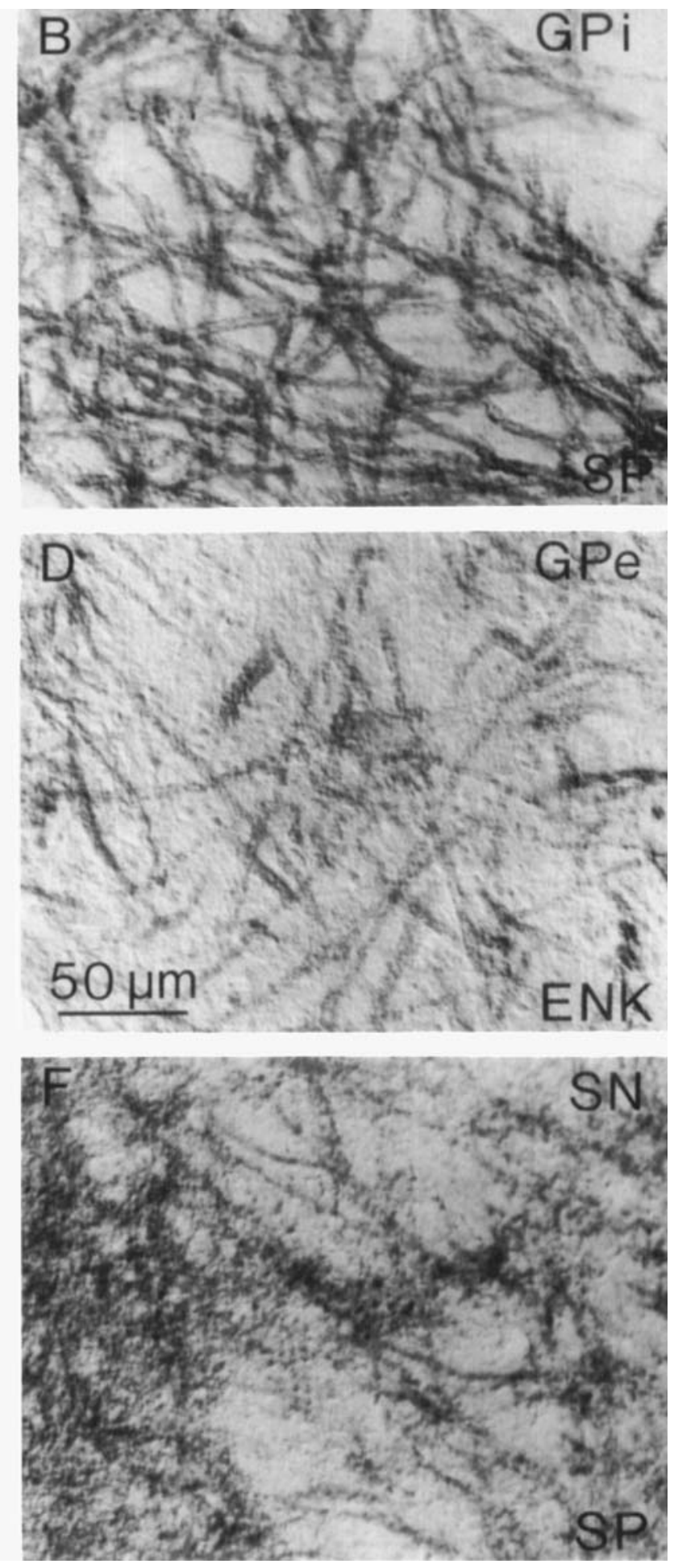

with the control subject, whereas the density of SP-immunoreactive fibers is comparable with the control subject. Comparison of fibers labeled for $S P$ in the nigra of the presymptomatic subject and control subject indicate a slight reduction in fiber abundance in the nigra of the preszmptomatio subject. 
density in the GPe was $72.8 \%$ of control; SP-IR fiber density in the $\mathrm{SN}$ was $88.9 \%$ of control. The variance of fiber abundance in control specimens was quite small; the standard deviations of SP-IR fiber abundance in the GPi was $4.8 \%$ and that of ENK-IR fiber abundance in the GPe was $11 \%$. Threshold analysis of the previously reported presymptomatic case and controls gave very similar results. SP-IR fiber density in the GPi was $94.5 \%$ of control; ENK-IR fiber density in the GPe was $76.5 \%$ of control; SP-IR fiber density in the $\mathrm{SN}$ was $86.9 \%$ of control. The standard deviation of ENK-IR fiber abundance in control GPe was $1.7 \%$ and of SP-IR fiber abundance in control GPi was $1.9 \%$.

\section{Discussion}

The immunohistochemical findings in this presymptomatic carrier of the HD allele are similar to those previously documented in pathologically verified early grade HD and similar to that in a prior subject with presymptomatic HD $[14,16]$. These results provide further confirmation that degeneration of striatal neurons projecting to the GPe occurs earlier in the course of $\mathrm{HD}$ than loss of neurons projecting to the GPi.

The available clinical information does not allow certain assessment of the subject's clinical status. The history of personality changes would be typical of early $\mathrm{HD}$, though such changes often occur years in advance of a clinically apparent movement disorder [20]. No family member noted any adventitious movements or impairment of coordination, and no family member felt that the subject had chorea. The history of difficulty in sitting still, however, is suggestive of subtle chorea. The clinical criteria for diagnosis of $\mathrm{HD}$ require the presence of an unequivocal movement disorder [20], and consequently, we classify this individual's condition as presymptomatic. Even if the subject had chorea and met criteria for a diagnosis of HD, the significance of the findings in this case would be undiminished. The absence of striatal cell loss and gliosis on routine neuropathological examination indicates that the subject would be classified as a grade zero in the pathological grading scheme of Vonsattel and colleagues [21], and still represent one of the earliest cases yet examined for striatal projection neuron changes.

Our conclusion of preferential loss of striato-GPe projection neurons is an inference based on changes in the pattern of staining of striatal projection neuron terminals. The perikarya of striatal neurons are difficult to reliably visualize with immunohistochemical procedures. Our method of analysis may actually underestimate the degree of striatal projection neuron terminal loss. We do not correct for any shrinkage of the pallidum or nigra. The presence of shrinkage (see Fig 1) would tend to increase fiber density and our fiber abundance measurements may consequently underestimate the degree of loss of striatal projection reuron termi- nals. Shrinkage effects are of especial importance in analyzing nigral SP-IR fibers. In our prior studies of patients with $\mathrm{HD}$ and presymptomatic $\mathrm{HD}$, changes in nigral SP-IR fiber density were accompanied by reduction of the area of the nigra containing SP-IR fibers. In this presymptomatic specimen, there were only subtle changes in either nigral SP-IR area or nigral SP-IR fiber density, suggesting that in this individual, striatonigral projection neurons are less affected than striato-GPe projection neurons. It is possible that the degeneration of striato-GPe neurons begins before the degeneration of striatonigral neurons. Although our immunohistochemical results most likely reflect the degeneration of striatal projection neurons, we cannot exclude the possibility that the perikarya of striato-GPe and striatonigral projection neurons are intact but have lost the ability to synthesize these neuropeptides.

In conclusion, we have shown changes in striatoGPe projection neurons in a presymptomatic carrier of the HD allele that are consistent with our previous data indicating preferential and presymptomatic loss of striato-GPe and striatonigral projection neurons in HD.

This work was supported by NS01300 (R.L.A.), NS19613 (A.B.Y. and J.B.P.), NS19620 (A.R.), NS28721 (A.R.), The Hereditary Disease Foundation (K.D.A.), The Neuroscience Center for Excellence of the University of Tennessee (K.D.A.), and the Huntington's Disease Society of America (L.S.D.).

We are very grateful to the family of this subject for their cooperation and support. We also thank Dr Charles Harlan, Davidson County (TN) Medical Examiner for his cooperation in the performance of the autopsy. We thank Ellen Karle and Betty Cook for their excellent technical assistance.

\section{References}

1. Ferrante RJ, Kowall NW, Beal MF, et al. Selective sparing of a class of striatal neurons in Huntington's disease. Science 1985; 230:561-564

2. Dawbarn D, Dequidt ME, Emson PC. Survival of basal ganglia neuropeptide $\mathrm{Y}$-somatostatin neurones in Huntington's disease. Brain Res 1985;340:251-260

3. Ferrante RJ, Beal MF, Kowall NW, et al. Sparing of acetylcholinesterase-containing striatal neurons in Huntington's disease. Brain Res 1987;411:162-166

4. Hirsch EC, Graybiel AM, Hersh LB, et al. Striosomes and extrastriosomal matrix contain different amounts of immunoreactive choline acetyltransferase in the human striatum. Neurosci Lett $1989 ; 96: 145-150$

5. Ferrante RJ, Kowall NW, Richardson EP, et al. Topography of enkephalin, substance $P$ and acetylcholinesterase staining in Huntington's disease striatum. Neurosci Lett 1986;71:283-288

6. Spokes EGS. Neurochemical alterations in Huntington's disease. Brain 1980;103:179-210

7. Beckstead RM, Cruz CJ. Striatal axons to the globus pallidus, entopeduncular nucleus and substantia nigra come mainly from separate cell populations in cat. Neuroscience 1986;19: $147-158$ 
8. Parent A, Bouchard C, Smith Y. The striatopallidal and striatonigral projections: two distinct fiber systems in primate. Brain Res 1984:303:385-390

9. Parent $A$, Smith $Y$, Filion $M$, et al. Distinct afferents to internal and external pallidal segments in the squirrel monkey. Neurosci Lett 1989;96:140-144

10. Feger J, Crossman AR. Identification of different subpopulations of neostriatal neurones projecting to globus pallidus or substantia nigra in the monkey: a retrograde fuorescence double labelling study. Neurosci Lett 1984;49:7-12

11. Gimenez-Amaya JM, Graybiel AM. Compartmental origins of the striatopalidal projection in the primate. Neuroscience $1990 ; 34: 111-126$

12. Graybiet AM. Neurotransmitters and neuromodulators in the basal ganglia. Trends Neurosci 1990;13:244-254

13. Reinet A, Anderson KD. The patterns of neurotransmitter and neuropeptide co-occurrence among striatal neurons. Conclusions based on recent findings. Brain Res Rev 1990;15:251-265

14. Reiner A, Albin RL, Anderson KD, et al. Differential loss of striatal projection neurons in Huntington disease. Proc Nat Acad Sci USA 1988;85:5733-5737
15. Albin RL, Young AB, Penney JB. The functional anatomy of basal ganglia disorders. Trends Neurosci 1989;12:366-375

16. Albin RL, Young AB, Penney JB, ct al. Abnormalitics of striatal projection neurons and $N$-methyl-1)-aspartate receptors in prisymptomatic Huntington's discasc. N Engl I Mod 1960;32?: $1293-1298$

17. Larhrop GM, Lalouel J-M, Julier C, et al. Multilocus linkage in humans: detection of linkage and estimation of recombination. Am J Hum Genet 1985;37:482-498

18. Mize RR, Holdefer RN, Nabors LB. Quantitative immunocytochemistry using an image analyzer. I. Hardware evaluation, image processing, and data analysis. J Neurosci Merhods 1988; $26: 1-24$

19. Haber SN, Nauta WIH. Ramifications of the ylobus pallidus in the rat as indicated by patteros of immunotustochemistry. Neuroscience 1983:9:245-260

20. Folstein SE. Huntington's disease: a disorder of families. Baltimore: Johns Hopkins Universiry, 1990

21. Vonsattel J-P, Myers RH, Stcvens TJ, et al. Neuropathological classification of Huntington's disease. J Neuropathol Exp Neurol $1985 ; 44: 559-577$ 